\title{
Treatment of severe tricuspid regurgitation with placement of percutaneous edge-to-edge posteroseptal and anteroseptal leaflet clips
}

\author{
Adam Rdzanek, Arkadiusz Pietrasik, Janusz Kochman, Piotr Scisło \\ $1^{\text {st }}$ Department of Cardiology, Medical University of Warsaw, Warsaw, Poland
}

Adv Interv Cardiol 2019; 15, 4 (58): 495-496 DOI: https://doi.org/10.5114/aic.2019.90228

The presence of secondary tricuspid regurgitation (TR) is considered to be an independent risk factor of an unfavorable clinical outcome in patients undergoing left heart valve disease interventions. In recent years, the MitraClip (Abbott, USA) system, originally developed for mitral regurgitation (MR) treatment, has proved to be effective in severe TR reduction in a selected population of patients. However, several anatomic features can hamper MitraClip utilization in this clinical setting. First of all, the presence of a pacemaker lead, causing reduced leaflet mobility, might prevent successful leaflet grasping. Secondly, the regurgitation jet location in the posteroseptal or anteroposterior coaptation line, partially due to the limited echocardiographic visualization of this area, is known to be a predictor of procedural failure during percutaneous edge-to-edge TR treatment. The present case exemplifies a successful solution of this technically demanding anatomic problem.

An 83-year-old woman with severe symptomatic secondary MR $\left(E R O=0.43 \mathrm{~cm}^{2}\right.$; MR vol. $\left.48 \mathrm{ml}\right)$ was scheduled for MitraClip therapy. The preprocedural echocardiographic examination also showed the presence of severe TR (VC-2D $8 \mathrm{~mm}$; VCA-3D $1.1 \mathrm{~cm}^{2}$ ) which was exacerbated due to the presence of a pacemaker lead causing partial posterior leaflet immobilization. The TR jet originated from both the posteroseptal and the anteroseptal coaptation line (Figure $1 \mathrm{~A}$ ).
Mitral valve repair was carried out according to the standard procedure. The implantation of two MitraClip devices led to a significant reduction of MR jets (MR-ERO $0.14 \mathrm{~cm}^{2}$ ). After the retrieval of a steerable sheath to the right atrium, tricuspid valve edge-to-edge repair was attempted. Under three-dimensional transesophageal echocardiographic (TEE-3D) guidance, the first clip was placed above the posteroseptal coaptation and orientated perpendicularly to the coaptation line. The pacemaker lead was pushed from the posterior leaflet toward the posteroseptal commissure. Once the clip was advanced to the right ventricle, an X-plane view based on TEE-3D, showing the precise device and leaflet orientation, allowed proper leaflet grasping and pacemaker lead entrapment between the clip and commissure. The second clip, implanted in a similar way between the anterior and septal leaflet close to the center of the valve, caused a further reduction of the regurgitation jet. The final TR parameters were as follows: VC-2D $4 \mathrm{~mm}$; VCA-3D $0.4 \mathrm{~cm}^{2}$, mean gradient $3 \mathrm{~mm} \mathrm{Hg}$ (Figure $1 \mathrm{~B}$ ). Transthoracic echocardiography carried out on the second day following the procedure showed a very good result of placement of both mitral and tricuspid clips (Figure $1 \mathrm{D}$ ) with only mild MR and mild to moderate TR presence.

The presented case describes, to the best of our knowledge, the first successful simultaneous posteroseptal and anteroseptal clip placement during complete percutaneous edge-to-edge tricuspid valve repair carried out in Poland. 

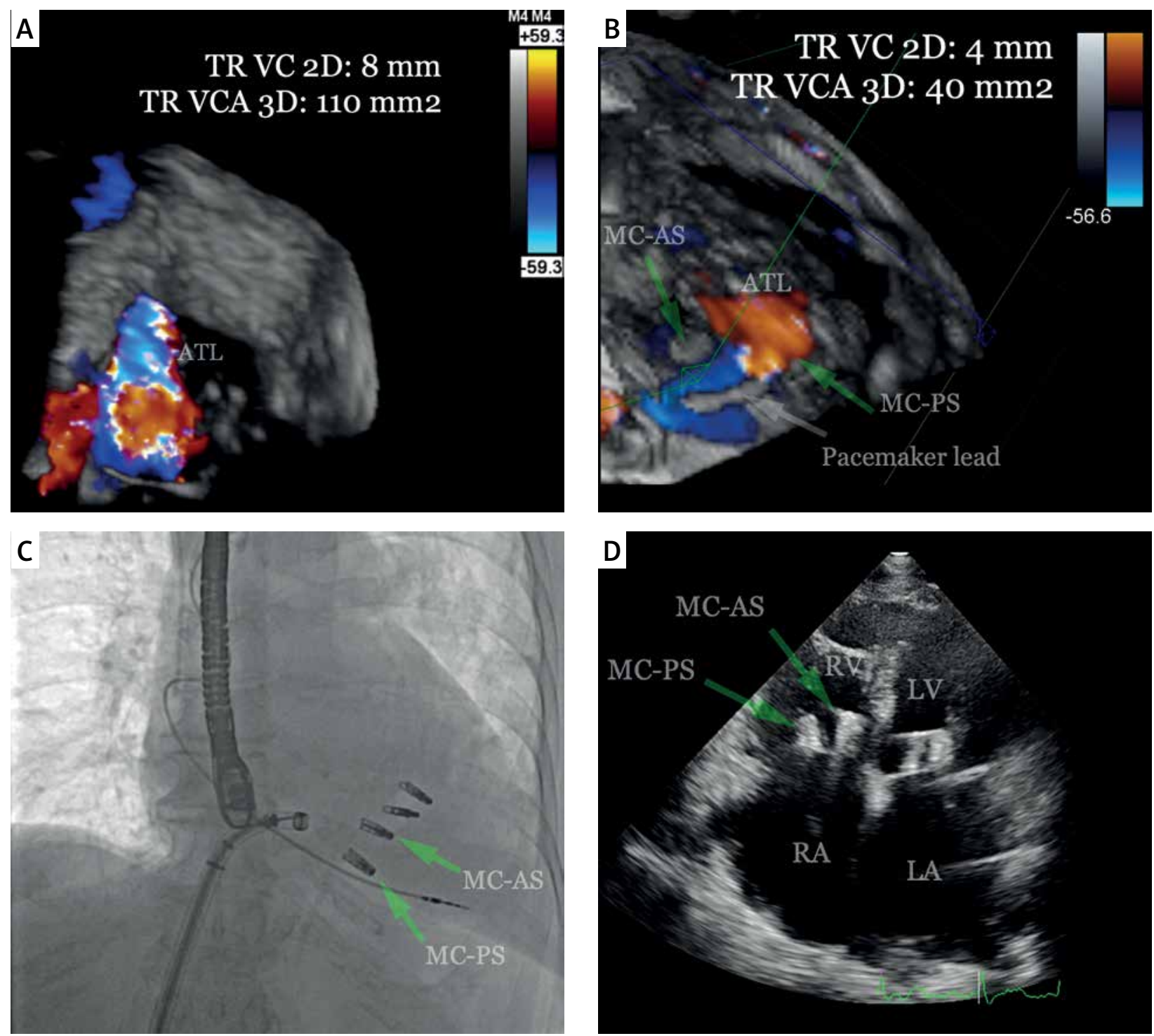

Figure 1. A - Baseline tricuspid regurgitation assessment. TEE-3D, mid-esophageal tricuspid valve planar view showing severe tricuspid regurgitation. B - Tricuspid regurgitation after implantation of two MitraClips (arrows) between posteroseptal (MC-PS) and anteroseptal (MC-AS). TEE-3D, mid-esophageal tricuspid valve planar view shows significant reduction of tricuspid regurgitation. C - Fluoroscopy showing position of mitral and tricuspid clips and position of pacemaker lead. D - Follow-up TEE-2D AP4C. Position of mitral and tricuspid clips 